(C) 2013. This manuscript version of Maximal Cell Density Predictions for compressible Polymer Foams is made available under the CC-BY-NC-ND 4.0 license http://creativecommons.org/licenses/by-nc-nd/4.0/

This document is the accepted manuscript version of a published article. Published by Elsevier in the journal " Published by Elsevier in the journal "Polymer" volume 54, issue 2. doi:10.1016/j.polymer.2012.11.067.

\title{
Maximal cell density predictions for compressible polymer foams
}

\author{
Yeongyoon Kim \\ Department of Physics and Astronomy, University of Waterloo, 200 University Avenue \\ West, Waterloo, Ontario, Canada N2L 3G1 \\ Waterloo Institute for Nanotechnology, University of Waterloo, 200 University Avenue \\ West, Waterloo, Ontario, Canada N2L 3G1 \\ Chul B. Park \\ Microcellular Plastics Manufacturing Laboratory, Department of Mechanical and Industrial \\ Engineering, University of Toronto, 5 King's College Road, Toronto, Ontario, Canada M5S \\ $3 G 8$ \\ P. Chen \\ Department of Chemical Engineering, University of Waterloo, 200 University Avenue West, \\ Waterloo, Ontario, Canada N2L 3 G1 \\ Waterloo Institute for Nanotechnology, University of Waterloo, 200 University Avenue \\ West, Waterloo, Ontario, Canada N2L $3 G 1$ \\ Russell B. Thompson \\ Department of Physics and Astronomy, University of Waterloo, 200 University Avenue \\ West, Waterloo, Ontario, Canada N2L 3G1 \\ Waterloo Institute for Nanotechnology, University of Waterloo, 200 University Avenue \\ West, Waterloo, Ontario, Canada N2L 3G1
}

\begin{abstract}
Thermodynamic upper bounds for polymer foam cell densities are predicted using compressible self-consistent field theory. It is found that the incompressible limit always gives the highest, and therefore ultimate, upper bound. Qualitative comparisons between the compressible and incompressible cases agree, indicating that low temperatures and high blowing agent content should be used to achieve high cell densities. The inhomogeneous bubble structure reveals deviations from the expected homogeneous Sanchez-Lacombe equation of state, consistent with some experimental results. A generalized Sanchez-Lacombe equation of state is discussed in the context of its suitability as a simple alternative to the Simha-Somcynsky equation of state.
\end{abstract}

Email address: thompson@uwaterloo.ca (Russell B. Thompson)

Preprint submitted to Elsevier

November 13, 2012 


\section{Introduction}

Polymer foams have found many applications in fields such as transportation, packaging, construction, consumer goods and many other areas [1]. New applications continue to be found, and there is particular economic and technological interest in advanced, high quality foams. These typically have bubble sizes on the order of microns or nanometers and can have superior properties compared to conventional polymer foams. The creation of such advanced micro- or nano-cellular foams is, however, a non-trivial task and many different techniques and chemistries are being explored to this end. To rationalize and reduce the scope of this search, it is desirable to have a method of determining whether a given combination of polymer and blowing agent under particular thermodynamic conditions is likely to be able to produce a foam of a desired quality, independent of the technique used to generate the foam. Such a method would not guarantee that the desired foam could necessarily be achieved, but it could provide evidence that a foam of a prescribed cell density is at least thermodynamically possible for given choices of polymer and blowing agent.

Recently, we introduced a method of predicting the maximum cell density of foams based on self-consistent field theory (SCFT) [2]. The method didn't predict an absolute cell density, but rather provided an upper bound that, from a thermodynamic perspective, real foams would be unlikely to exceed. One would expect that actual foams would in fact have cell densities significantly lower than the predicted maximum, due to important kinetic and processing issues. Polymer foaming is after all a non-equilibrium process and the SCFT method is an equilibrium model. Nonetheless, one can use SCFT for polymer foaming as has been argued in references [3] and [4]. Our method was not quantitative however but rather provided only qualitative guidance such as finding that foaming should take place at as low a practical temperature as possible, that the maximum amount of blowing agent that doesn't cause spinodal decomposition should be used, and that polymer surface tension is not always an important factor in predicting cell densities [2]. The root of the qualitative nature of our predictions was that we used, for simplicity, an incompressible model of polymer foaming. This is far from realistic, but there is evidence that such an approach can be used to give reliable qualitative predictions $[5,6,7]$.

In this paper, we drop the incompressible limitation in order to explore the extent to which compressibility affects our previous qualitative predictions. We find that all our previous predictions continue to be valid and, what's more, that the incompressible case does indeed set the thermodynamic upper bound on polymer foam cell densities. In addition, we are able to comment on the limitations of the Sanchez-Lacombe equation of state and we speculate on how it might be easily adapted to become competitive with the Simha-Somcynsky equation of state. The results we present in this work are a qualitative demonstration of the approach, but the methodology can be made quantitative under certain conditions. 


\section{Theory}

We describe a compressible mixture of monodisperse polymer and either gas or super-critical fluid using a hole-based self-consistent field theory (SCFT). In hole theories, a compressible formalism is achieved by representing voids in the mixture as particles that occupy some space. These fictitious particles have no energetic interactions with any of the true chemical species but contribute only translational entropy to the problem. An appropriate choice of the volume for the hole particles allows this entropy to produce the experimentally expected equation of state (EOS) - more holes correspond to lower pressure and fewer holes correspond to higher pressure. The hole-based SCFT that we use in this work was first introduced by Hong and Noolandi [8]. As there are many very good reviews of SCFT, such as references [9] and [10], we will not present the details of SCFT here but rather refer the reader to our previous works for incompressible $[2,3]$ and compressible $[6,11]$ polymer foams. The SCFT model for a compressible polymer solvent system can however be summarized by the free energy functional

$$
\begin{aligned}
\tilde{\mathcal{F}} \equiv \frac{N F}{\rho_{0} k_{B} T V}= & -\phi_{p} \ln \left(\frac{Q_{p}}{V \phi_{p}}\right)-\frac{\phi_{s}}{\alpha_{s}} \ln \left(\frac{Q_{s}}{V \phi_{s}}\right)-\frac{\phi_{h}}{\alpha_{h}} \ln \left(\frac{Q_{h}}{V \phi_{h}}\right) \\
& +\frac{1}{V} \int d \mathbf{r}\left[\chi_{p s} N \varphi_{p}(\mathbf{r}) \varphi_{s}(\mathbf{r})+\frac{1}{2} \chi_{p p} N \varphi_{p}(\mathbf{r}) \varphi_{p}(\mathbf{r})\right. \\
& +\frac{1}{2} \chi_{s s} N \varphi_{s}(\mathbf{r}) \varphi_{s}(\mathbf{r})-w_{p}(\mathbf{r}) \varphi_{p}(\mathbf{r})-w_{s}(\mathbf{r}) \varphi_{s}(\mathbf{r}) \\
& \left.-w_{h}(\mathbf{r}) \varphi_{h}(\mathbf{r})\right] .
\end{aligned}
$$

In equation (1), $\varphi_{p}(\mathbf{r}), \varphi_{s}(\mathbf{r})$ and $\varphi_{h}(\mathbf{r})$ are the local (position dependent) volume fractions of polymer, gas (or fluid), and holes, respectively. The subscript " $s$ " is used for the gas volume fraction since it is customary in SCFT to refer to molecules lacking polymeric internal degrees of freedom as "solvent" molecules. We will continue to use this term in this paper. The total system volume fractions for polymer, solvent and holes are $\phi_{p}, \phi_{s}$ and $\phi_{h}$, respectively, and single molecule partition functions are given by $Q_{p}, Q_{s}$ and $Q_{h}$, respectively. The ratio of the volume of a solvent molecule to a polymer molecule is denoted by $\alpha_{s}$ and that of a hole to a polymer molecule is $\alpha_{h}$. The volume of one polymer segment is designated as $\rho_{0}^{-1}$ and the degree of polymerization is $N$. The segregation between polymer segments, solvent molecules and holes would normally be specified using Flory-Huggins parameters $\chi_{p s}, \chi_{p h}$ and $\chi_{s h}$, but we find this misleading as the holes don't actually interact with polymer or solvent. Instead, we follow our previous convention [11] and use instead the parameters $\chi_{p s}, \chi_{p p}$ and $\chi_{s s}$ for polymer-solvent, polymer-polymer and solvent-solvent interactions, respectively. These parameters are related to the chemistry of the polymer and solvent molecules and are also inversely proportional to temperature $T$. Following reference [11], we assume a dimensionless temperature dependence for each $\chi$ (or more conveniently, the product $\chi N$ ) of

$$
\chi N=\frac{A}{T}+B
$$


where we chose $A_{p s}=270, A_{p p}=-12, A_{s s}=0$ and all $B$ 's as zero. The mean fields felt by each polymer segment, solvent molecule or hole due to interactions with all other segments, molecules and holes in the system are given by $w(\mathbf{r})_{p, s}$ or $h$. The left hand side of (1) is the system free energy $(F)$ per system volume $(V)$, made dimensionless using appropriate factors. In this work, all lengths are phrased in terms of the radius of gyration of a polymer, $R_{g}$. Variation of equation (1) with respect to all functions yields a set of coupled, non-linear equations to be solved self-consistently. The hole formalism allows one to complete the set of equations with the expression

$$
\varphi_{p}(\mathbf{r})+\varphi_{s}(\mathbf{r})+\varphi_{h}(\mathbf{r})=1 .
$$

The SCFT equations are solved numerically as described in previous work [3, $6,7,11]$. For any given hole volume fraction and box size, the sample pressure can be calculated using the formula

$$
P=-\frac{\partial F}{\partial V}{ }_{n_{p}, n_{s}, T}
$$

where $n_{p}$ and $n_{s}$, the number of polymer and solvent molecules in the volume $V$, are held constant.

The formalism is used in a way that parallels reference [2]. We choose closepacked polymer and solvent volume fractions for the incompressible case, $\phi_{p}^{0}$ and $\phi_{s}^{0}$, and change the size of the calculational volume $V$ to find the maximum cell density. The definition of the cell density is given in the next paragraph. We then add holes by increasing the hole volume fraction $\phi_{h}$ from zero. This means that $\phi_{p}$ and $\phi_{s}$ will have to change for equation (3) to be satisfied, but the ratio of $\phi_{p}$ to $\phi_{s}$ must remain the same as the ratio $\phi_{p}^{0}$ to $\phi_{s}^{0}$. The volume $V$ is varied again to find the maximum cell density for this choice of $\phi_{h}$. This procedure is repeated for a range of hole volume fractions, in other words, for a range of sample volumes.

The calculation is done in spherical coordinates with only one bubble in the volume $V$, so the bubble will naturally centre itself within the spherical coordinates in order to minimize the free energy [12]. This is depicted schematically in figure 1(a) with a data plot of a radial cross section of the system shown in figure 1(b). The volume $V$ is then the average volume per bubble. The typical cell number density is thus the inverse of $V$, at least in principle. One can choose to define the cell density as the number of cells per unit volume or the number of cells per unit volume of polymer [13]. We chose the latter in reference [2] and we follow this convention again in this work. Therefore the cell density is defined as the inverse of the product of the calculational box volume times the overall polymer volume fraction. This gives us the best possible cell density for a given amount of solvent and holes. Our method is not able to account for kinetic phenomena, bubble polydispersity and other experimental realities, so our predictions must be viewed as upper limits on possible cell densities. The maximum cell density will typically be found at the smallest possible bubble radius for any given $\phi_{s}$ and $\phi_{h}$, that is, at the critical radius. Paralleling our 


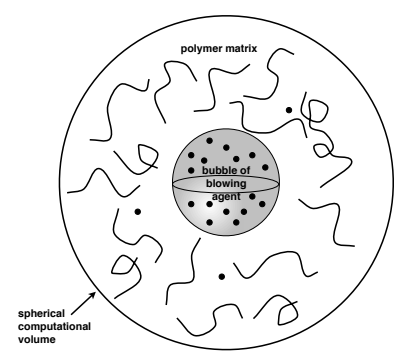

(a)

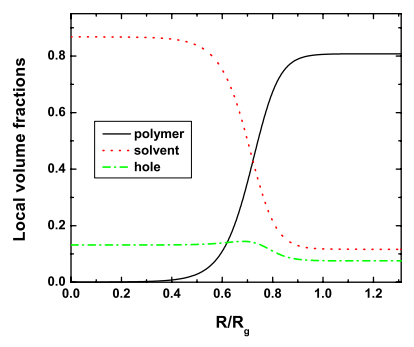

(b)

Figure 1: (a) A schematic of the system. A spherical computational box is used containing a centred single bubble of fluid surrounded by polymer. (b) A radial cross section of the spherical computational volume containing a single bubble using data from an SCFT calculation.

previous article however, one can also find maximum cell densities for other criteria such as the cell density corresponding to the maximum amount of bubble surface area per volume of foam or to the smallest radius cell that completely excludes polymer from its interior. Specifically, the first of these cases, the critical radius of the bubble, is considered to be the smallest radius that our SCFT computation predicts is stable. Smaller than this radius, the bubble structure will collapse during computation and a uniform mixture is predicted. For the second case, the surface area of a bubble can be found given the bubble radius. We give an SCFT definition of bubble radius in a previous work [3], and this allows us to predict the bubble area for a given calculation volume, specifically, the bubble area to volume ratio. The radius of this maximum is, surprisingly, not necessarily the critical radius. Thirdly, the local volume fractions of each chemical species are outputs of the SCFT, so it is straightforward to examine the volume fraction of polymer at the centre of the bubble as a function of bubble radius. The smallest radius at which there is negligible polymer at the bubble center is different from both the critical radius and the maximum bubble area radius. We examine all three of these cases in the Results and Discussion section. The model can be made quantitative with appropriate input data, but since the predictions are for maximal cell densities rather than actual cell densities, we here view trends to be more important than specific values.

\section{Results and Discussion}

For our SCFT calculations, we examined two dimensionless temperatures (using equation (2)) $T=1.5$ and $T=2.0$ for molecular sizes described by $\alpha_{h}=\alpha_{s}=0.01$. For $T=1.5$, we took $\phi_{p}^{0}=0.74, \phi_{s}^{0}=0.26, \chi_{p p} N=-8$, $\chi_{s s} N=0$, and $\chi_{p s} N=180$. For $T=2.0$, we took $\phi_{p}^{0}=0.69, \phi_{s}^{0}=0.31$, $\chi_{p p} N=-6, \chi_{s s} N=0$, and $\chi_{p s} N=135$. These values were chosen so that 


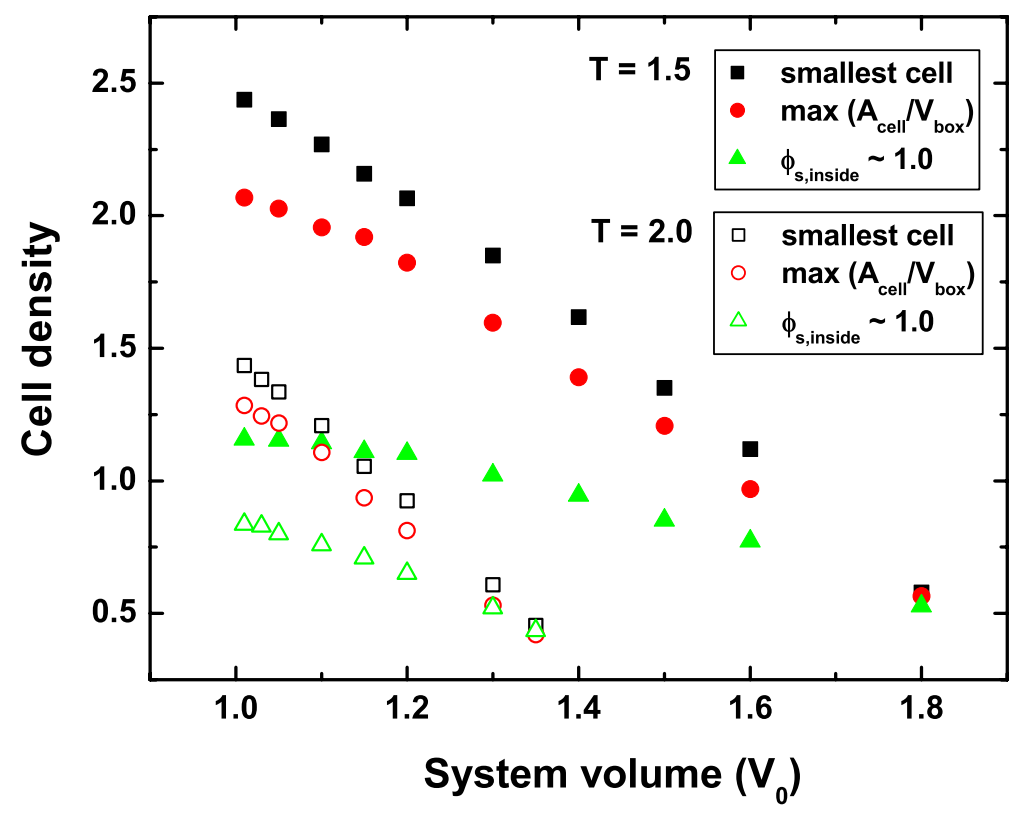

Figure 2: Cell densities as a function of sample volume. For dimensionless temperature 1.5, the black squares show the smallest cell possible, that is, the critical radius. The red circles show the maximum bubble area to volume ratio and the green triangles give the highest cell density for which there is almost no polymer inside the centre of the bubble. Open black squares, open red circles and open green triangles give the same respective quantities for dimensionless temperature 2.0.

the two different temperatures would have the same pressure when in an initial homogeneous (high pressure, approaching incompressible) state. These $\chi$ parameters correspond to a blowing agent of a "nitrogen" type, since we get higher solubilities at higher temperatures [14]. Other choices of $\chi$ parameters can give lower solubilities at higher temperatures which would correspond to "carbon dioxide" type blowing agents $[15,16,17,18,19]$. Figure 2 shows cell densities as a function of sample volume for the above choices of parameters corresponding to $T=1.5$ and $T=2.0$. The sample volume is presented rather than sample pressure since the foaming sample will not be in equilibrium with the experimental ambient pressure and therefore the actual sample pressure may be more difficult to determine than the sample volume. In figure 2 , the two different temperatures are presented for three different situations. These three situations are: the cell density at the critical radius, the cell density when the bubble area to system volume ratio is maximum, and the cell density for 


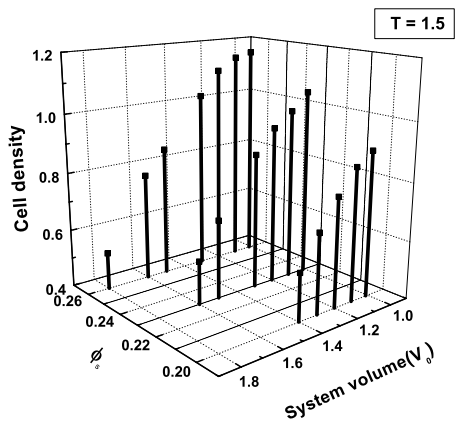

(a)

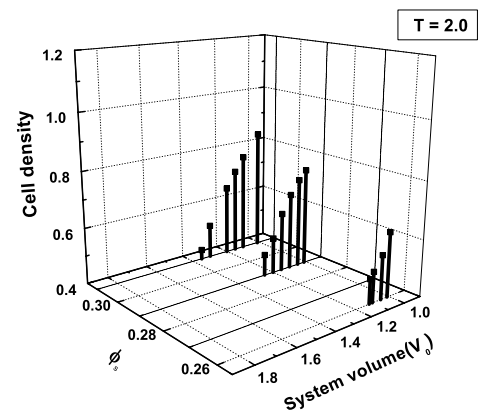

(b)

Figure 3: Cell densities as functions of close-packed solvent (blowing agent) volume fraction and sample volume. Cell densities are determined assuming polymer volume fraction within a bubble is zero. Results for other cases, specifically bubble surface area to sample volume ratio and critical radius, are qualitatively the same. (a) $T=1.5$. (b) $T=2.0$.

the smallest bubble that completely excludes polymer from its centre. For both dimensionless temperatures, $T=1.5$ and $T=2.0$, and for all three cases, we find that the maximum cell density corresponds to the incompressible system, normalized as $V=1.0 V_{0}$.. These results lead immediately to two important validations of the incompressible model [2]. Firstly, the incompressible system does indeed give the overall maximal cell density for a given polymer foam system. Therefore, more complicated formalisms incorporating various EOS are not necessary for establishing, qualitatively, trends in maximum cell density behaviour. Secondly, cell densities at $T=1.5$ are always higher, for all situations examined here, than for $T=2.0$. Therefore, our previous finding that foaming should be performed at the lowest possible practical temperatures in order to maximize cell densities is born out in the compressible case. Again, for qualitative comparisons, the simpler, incompressible formalism can therefore be used. The two panels of figure 3 show maximum cell densities as functions of both sample volume and overall solvent volume fraction. The first panel is for $T=1.5$ and the second panel is for $T=2.0$. For both temperatures, we show the case of complete polymer exclusion from the interior of a bubble, but the results are qualitatively the same for the critical radius and maximum bubble area to volume ratio conditions on the cell density. These figures confirm a third result of the incompressible model, that is, that cell densities are increased with increasing solvent volume fraction. Therefore, one should maintain a high blowing agent content, but no so high as to cause spinodal decomposition. In general then, one wants to keep the sample from expanding as much as possible while the bubbles grow, that is, as close to incompressible as possible. In all cases, this gives the highest cell density. For example, when dropping pressure to cause foaming, the lower pressure at which the foaming takes place might be kept as high as possible. In figure 3 we also show cell density data out to 
higher sample volumes for high $\phi_{s}^{0}$ than for low $\phi_{s}^{0}$. This is because the bubble structure in SCFT breaks down at different values of sample volume depending on $\phi_{s}^{0}$. This is evidence that foams with more blowing agent may be capable of greater expansion than foams with less blowing agent.

While the upper bound cell density corresponds to the incompressible case, one will often want information about foams which depends critically on the particular EOS that is applicable. The Hong and Noolandi SCFT that we are using can be made to reduce to the Sanchez-Lacombe (SL) EOS [8], but it does not necessarily reduce to SL. This is important since the SL EOS has been shown to fail for polymer foams under certain circumstances [17, 20]. The Simha-Somcynsky (SS) EOS has been found to be quantitatively superior for polymer foams $[17,20]$. On the other hand, the SS EOS is much more complicated and has more free parameters than SL [21], so it's not surprising that better agreement can be found. Figure 4 shows pressure as a function of sample volume for both homogeneous (SL) and inhomogeneous polymer (bubble SCFT) systems. It demonstrates that inhomogeneity alone is enough to cause disagreement between SL and inhomogeneous SCFT predictions. While the SL and SCFT EOS lines seem to fall close to each other in panel (a), the blow up in panel (b) at high pressures reveals quite a difference. At lower pressures in panel (c), the difference is reduced, but still exists. Inhomogeneity is not the cause of the disagreement between experiment and theory in references [17] and [20] because these works examine solubilities, not foaming. However, our results show that inhomogeneity causes increasing disagreements with the standard SL EOS with increasing pressures. This parallels the aforementioned solubility experiments that also predict the failure of SL for high pressures [17, 20, 22]. We further notice that this deviation from SL is larger at lower temperatures. To our knowledge, this has yet to be investigated experimentally. For the inhomogeneous results in figure 4 , we used bubbles that completely excluded polymer from the bubble center. For the critical radius and maximum bubble area to volume cases, the results are much less dramatic but qualitatively the same. Furthermore, SL assumes the same, lattice-derived, fixed molecular volume for polymer segments, solvent molecules and holes. While we have done the same here, we don't have to. These molecular volume values might depend on pressure or temperature themselves. Thus more parameters appear which, although not the same as the SS theory, can give the homogeneous EOS derived from SCFT a wider scope. In this work, for simplicity, we have used $\alpha_{s}=\alpha_{h}$ so the homogeneous version of equation (1) reduces to SL. For quantitative predictions, this condition could be relaxed. These sort of quantitative predictions and possible relationships between a generalized SCFT derived SL EOS and different modifications of the SL EOS, such as those suggested in references [23] and [24], are beyond the scope of this work.

\section{Conclusions}

We have generalized the SCFT approach of [2] to account for compressibility in the prediction of the maximum possible cell densities for polymer foams using 


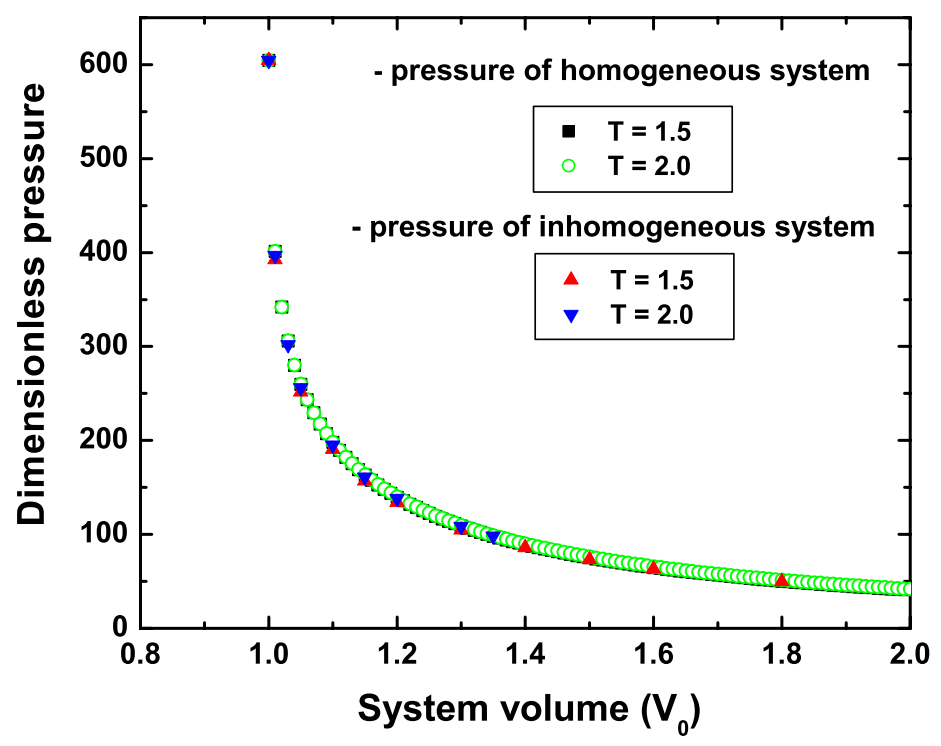

(a)

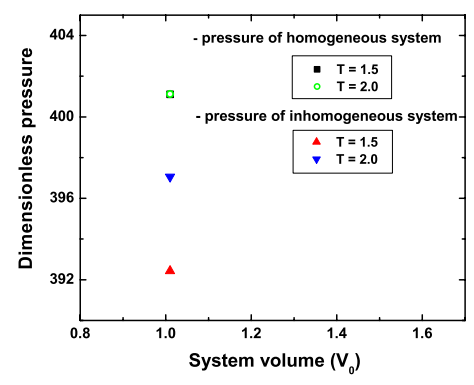

(b)

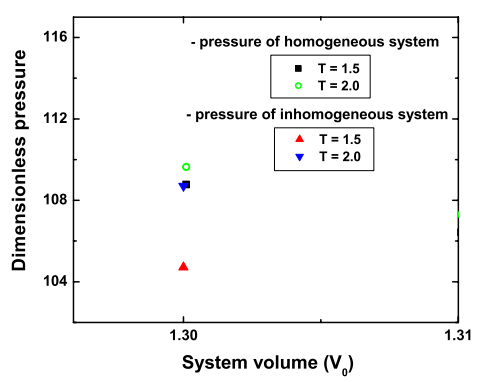

(c)

Figure 4: Dimensionless pressure as a function of sample volume. Panel (a) shows predictions for the homogeneous pressure for $T=1.5$ (black squares) and $T=2.0$ (green open circles). It also shows the inhomogeneous pressure for $T=1.5$ (red triangles) and $T=2.0$ (blue inverted triangles). Panels (b) and (c) show blow-ups at sample volumes of $1.01 V_{0}$ and $1.3 V_{0}$. 
the hole theory of Hong and Noolandi [8]. We find that the upper limit cell density in all cases still corresponds to the incompressible case. This means that the simpler, incompressible formalism is appropriate for determining qualitative trends for composition, chemistry and temperature choices for maximizing cell densities in polymer foams. Consistent with reference [2], lower temperatures are predicted to give higher cell densities, not only for incompressible systems, but also all ranges of compressibility. Higher blowing agent content is also confirmed as favourable to high cell densities. To maximize cell densities, we find here that the system should be maintained as close to incompressible as possible, that is, that the sample volume expansion should be restrained as much as possible while bubbles are growing.

We report deviations in the EOS behaviour of our inhomogeneous system from that of the SL EOS at high pressures that parallel the experimental findings of references [17] and [20]. This is particularly notable since we chose our SCFT formalism to reduce to SL in the homogeneous limit. If the volumes of holes and solvents are chosen to be different however, a new parameter is added to the SL equation, giving a generalized version that may be more competitive with Simha-Somcynsky quantitatively, while maintaining the essential simplicity and corresponding states principle of SL. SCFT therefore provides a facile route to deriving a generalized SL EOS. In future work, the relationship of this new SL EOS with other modified SL equations may be established and the quantitative predictive power established. The SCFT method may also be used to make predictions about nucleation barriers, expansion ratios, open versus closed cell foam structures and cell wall thicknesses.

\section{References}

[1] S.-T. Lee, C. B. Park, N. S. Ramesh, Polymeric Foams: Science and Technology, CRC Press, Boca Raton, 2007.

[2] Y. Kim, C. B. Park, P. Chen, R. B. Thompson, Towards maximal cell density predictions for polymeric foams, Polymer 52 (2011) 5622-5629.

[3] Y. Kim, C. B. Park, P. Chen, R. B. Thompson, Origins of the failure of classical nucleation theory for nanocellular polymer foams, Soft Matter 7 (2011) 7351-7358.

[4] D. W. Oxtoby, Density functional methods in the statistical mechanics of materials, Annu. Rev. Mater. Res. 32 (2002) 39-52.

[5] S. N. Leung, A. Wong, Q. Guo, C. B. Park, J. H. Zong, Change in the critical nucleation radius and its impact on cell stability during polymeric foaming processes, Chem. Eng. Sci. 64 (2009) 4899-4907.

[6] R. B. Thompson, J. R. MacDonald, P. Chen, Origin of change in molecularweight dependence for polymer surface tension, Phys. Rev. E 78 (2008) 030801-1-4. 
[7] R. B. Thompson, C. B. Park, P. Chen, Reduction of polymer surface tension by crystallized polymer nanoparticles, J. Chem. Phys 133 (2010) 144913$1-7$.

[8] K. M. Hong, J. Noolandi, Conformational entropy effects in a compressible lattice fluid theory of polymers, Macromolecules 14 (1981) 1229-1234.

[9] M. W. Matsen, Self-consistent field theory and its applications, in: G. Gompper, M. Schick (Eds.), Soft Matter Volume 1, Wiley-VCH, 2005, pp. 87-178.

[10] G. H. Fredrickson, The Equilibrium Theory of Inhomogeneous Polymers, Oxford University Press, New York, 2006.

[11] H. Park, R. B. Thompson, N. Lanson, C. Tzoganakis, C. B. Park, P. Chen, Effect of temperature and pressure on surface tension of polystyrene in supercritical carbon dioxide, J. Phys. Chem. B 111 (2007) 3859-3868.

[12] The assumption of a spherical bubble is common to this SCFT work and classical nucleation theory. This approximation should give a reasonable average prediction for cell density even though under many circumstances bubbles may be deformed.

[13] X. Xu, C. B. Park, D. Xu, R. Pop-Iliev, Effects of die geometry on cell nucleation of ps foams blown with $\mathrm{co}_{2}$, Polym. Eng. Sci. 43 (2003) 13781390 .

[14] One could choose to use $\chi_{p s}, \chi_{p h}$ and $\chi_{s h}$ instead of our choice of $\chi_{p s}$, $\chi_{p p}$ and $\chi_{s s}$. Converting from our notation to this more common notation reveals no negative $\chi$ parameters, as expected.

[15] J. G. Lee, R. W. Flumerfelt, Nitrogen solubilities in low-density polyethylene at high temperatures and high pressures, J. Appl. Polym. Sci. 58 (1995) 2213-22219.

[16] Y. Sato, M. Yurugi, K. Fujiwara, S. Takishima, H. Masuoka, Solubilities of carbon dioxide and nitrogen in polystyrene under high temperature and pressure, Fluid Phase Equilibria 125 (1996) 129-138.

[17] G. Li, J. Wang, C. Park, Investigating the solubility of $\mathrm{CO}_{2}$ in polypropylene using various eos models, Cell. Polym. 25 (2006) 237-248.

[18] G. Li, J. Wang, C. B. Park, R. Simha, Measurement of gas solubility in linear/branched pp melts, J. Polym. Sci. Pol. Phys. 45 (2007) 2497-2508.

[19] G. Li, F. Gunkel, J. Wang, C. B. Park, V. Altstädt, Solubility measurements of $\mathrm{n}_{2}$ and $\mathrm{co}_{2}$ in polypropylene and ethene/octene copolymer, J. Appl. Polym. Sci. 103 (2007) 2945-2953. 
[20] M. M. Hasan, Y. G. Li, G. Li, C. B. Park, P. Chen, Determination of solubilities of co2 in linear and branched polypropylene using a magnetic suspension balance and a pvt apparatus, J. Chem. Eng. Data 55 (2010) $4885-4895$.

[21] R. Simha, T. Somcynsky, On the statistical thermodynamics of spherical and chain molecule fluids, Macromolecules 2 (1969) 342-350.

[22] Y. G. Li, C. B. Park, The effects of branching on the pvt behaviors of $\mathrm{pp} / \mathrm{CO}_{2}$ solutions, Ind. Eng. Chem. Res. 48 (2009) 6633-6640.

[23] R. A. Krenz, T. Laursen, R. A. Heidemann, The modified sanchez-lacombe equation of state applied to polydisperse polyethylene solutions, Ind. Eng. Chem. Res. 48 (2009) 10664-10681.

[24] H. Machida, Y. Sato, R. L. Smith, Simple modification of the temperature dependence of the sanchezlacombe equation of state, Fluid Phase Equilibria 297 (2010) 205-209. 